\title{
Hypofractionated Accelerated Chemo-radiotherapy (Chemo-HypoAR) With Cisplatin and Liposomal Doxorubicin for the Treatment of Patients With Uterine Sarcomas
}

\author{
SPYROS DOMOXOUDIS, IOANNIS M. KOUKOURAKIS, \\ AXIOTIS G. GIAKZIDIS and MICHAEL I. KOUKOURAKIS \\ Department of Radiotherapy - Oncology, Medical School, \\ Democritus University of Thrace, Alexandroupolis, Greece
}

\begin{abstract}
Background/Aim: Uterine sarcoma is an aggressive tumor associated with poor survival, compared to endometrioid carcinoma. Postoperative local radiotherapy and chemotherapy are controversial. Patients and Methods: We report a retrospective analysis of 14 patients with uterine homologous type carcinosarcoma (9 patients) or leiomyosarcoma (5 patients), treated with postoperative $3 D$-conformal accelerated hypofractionated radiotherapy $(2.7 \mathrm{~Gy} /$ fraction for 14 fractions followed by one fraction of 6-8 Gy dose to the vagina). Chemotherapy with cisplatin $\left(50 \mathrm{mg} / \mathrm{m}^{2}\right)$ and liposomal doxorubicin $\left(20 \mathrm{mg} / \mathrm{m}^{2}\right)$, was also administered bi-weekly for two cycles before and for three cycles during radiotherapy. Results: Chemotherapy induced only grade 1 neutropenia or anemia in $4 / 14$ (28.5\%) and 5/14 (35.7\%) of patients, respectively. Two patients $(2 / 14,14.2 \%)$ interrupted their radiotherapy for one and two weeks, respectively, due to grade II persistent diarrhea. Within a median of 58 months (range $=8$ 137 months) of follow-up, none of the patients presented with loco-regional relapse. Two patients developed distant metastasis. Conclusion: Concurrent hypofractionated and accelerated chemo-radiotherapy (chemo-HypoAR) is feasible and provides excellent survival figures.
\end{abstract}

Uterine sarcoma is a histologically heterogeneous entity that comprises pure sarcomas, mixed sarcomas, mixed mesodermal tumors, unclassified sarcomas and malignant lymphomas (1). Leiomyosarcomas (LS) and mixed malignant mullerian tumors

This article is freely accessible online.

Correspondence to: Michael I. Koukourakis, PO BOX 12, Alexandroupolis 68100, Greece. Tel: +30 2551074622, Fax: +30 2551030349, e-mail: targ@her.forthnet.gr

Key Words: Uterine sarcoma, radiotherapy, cisplatin, liposomal doxorubicin, hypofractionation, acceleration. are considered to be the most frequent uterine sarcoma subtypes $(2,3)$. The diagnosis of a sarcoma is established after hysterectomy. Deep myometrial invasion or uterine serosa spread, node involvement that occurs in $15-30 \%$ of patients, adnexal involvement and extrapelvic metastasis are linked with poor prognosis of these patients (4-6).

Uterine sarcoma is considered an aggressive tumor. Survival of patients with uterine sarcoma is poorer than the one of patients with endometrioid adenocarcinoma; the 5year disease specific survival estimated to be around $50 \%$ (7). Local radiotherapy seems to reduce the risk of locoregional recurrence, although some non-randomized studies support that the role of radiotherapy is still unclear $(8,9)$. Distant metastasis is quite frequent. In a randomized trial by EORTC, local radiotherapy reduced the risk of locoregional recurrence from $24 \%$ to $12 \%$, but there was no impact on overall survival (10). The value of additional chemotherapy in improving overall survival is controversial $(6,11,12)$. A randomized trial conducted by the GOG, failed to show any benefit from postoperative chemotherapy (13). In a recent analysis, however, on 4,906 patients with carcinosarcoma treated with combined radiotherapy and chemotherapy, an improved survival was noted compared to patients receiving only one treatment modality (14).

In the current study we analysed our experience with treating patients with uterine sarcoma, using accelerated hypofractionated pelvic radiotherapy concurrently with chemotherapy (chemo-HypoAR), providing evidence of encouraging treatment outcomes.

\section{Patients and Methods}

Fourteen patients with sarcoma of the uterus treated with concurrent hypofractionated accelerated chemo-radiotherapy, between 2005-2016, are retrospectively analyzed. The age of patients ranged from 44-83 years (median 69). Histologically, nine $(9 / 12 ; 64.3 \%)$ were mixed malignant müllerian tumors (homologous type carcinosarcoma; CS) and five $(5 / 12 ; 64.3 \%)$ pure leiomyosarcomas. The FIGO stage of 
patients was as follows: Stage 1a (3 pts), $1 \mathrm{~b}$ (6 pts), 2 (4pts), 3b (1 pt). The median follow-up of patients ranged from 1-137 months (median 50 months). For patients alive, this ranged from 8-137 months (median 58 months).

Thirteen out of 14 patients underwent total abdominal hysterectomy and bilateral oophorectomy without lymphadenectomy, while one with stage IIIB disease was deemed inoperable. Four patients were treated with postoperative radiotherapy without chemotherapy, for medical reasons. The remaining 10 patients were treated with a concurrent chemo-radiotherapy protocol described below.

Radiotherapy was directed to the pelvis to include pelvic lymph nodes up to the level of lumbar 4 vertebrae and, down to include the whole vaginal area. A 3D-conformal radiotherapy technique was applied, using 4-fields (box). Radiotherapy was given with an $18 \mathrm{MV}$ linear accelerator endowed with a multileaf collimator. Fourteen fractions of 2.7 Gy were delivered within 19 days ( 5 fractions per week). One week later, a booster dose of 6-8 Gy in one fraction was applied to the vagina, using a 6-field technique (two lateral and four oblique fields). For one inoperable patient, following pelvic irradiation, two booster fractions of $6 \mathrm{~Gy}$ were applied to the uterus and gross tumor detectable in CT/MRI scans. The above external beam radiotherapy scheme delivers a Normalized Total Dose (NTD) to normal tissues of $42 \mathrm{~Gy}$ (for $\alpha / \beta=4 \mathrm{~Gy}$ ) within 19 days. A biologically equivalent conventionally fractionated radiotherapy scheme ( $2 \mathrm{~Gy} /$ fraction regimen) demands 21 days of therapy (30 days), thus our regimen reduced the overall radiotherapy external beam treatment time by 11 days. The radiobiological methods applied for calculation of the NTD have been previously reported $(15,16)$.

The chemo-radiotherapy regimen included 2 pre-radiotherapy biweekly-cycles of cisplatin $\left(50 \mathrm{mg} / \mathrm{m}^{2}\right)$ and liposomal doxorubicin $\left(20 \mathrm{mg} / \mathrm{m}^{2}\right)$. Two weeks after the $2 \mathrm{nd}$ cycle, radiotherapy started together with the same bi-weekly chemotherapy regimen throughout the radiotherapy course, to a total of 5 cycles.

Patients were followed with a CT-scan of the pelvis, upper abdomen and chest every six months for the first 3 years and yearly thereafter. Gynecological and cytological examination of the vaginal cuff was performed every year.

Ethical standards. All patients had given written informed consent and the protocol of accelerated hypofractionated chemoradiotherapy for pelvic tumors was approved by the local research ethics committee (SD 7/26-2-2004).

\section{Results}

The regimen was well tolerated by all patients. Chemotherapy induced only grade 1 neutropenia or anemia in 4 and 5 patients, respectively. No other chemotherapy-related toxicity was noted. Out of 14 patients, 12 accomplished the radiotherapy schedule without any delays, while two patients interrupted their therapy flux for 1 and 2 weeks, respectively, due to grade 2 persistent diarrhea.

Within 58 months of median follow-up, none of the treated patients presented with loco-regional relapse. Two patients developed distant metastasis, one of them (initially staged as inoperable 3b) 1 month and the other 18 months after the accomplishment of radiotherapy. None of these two
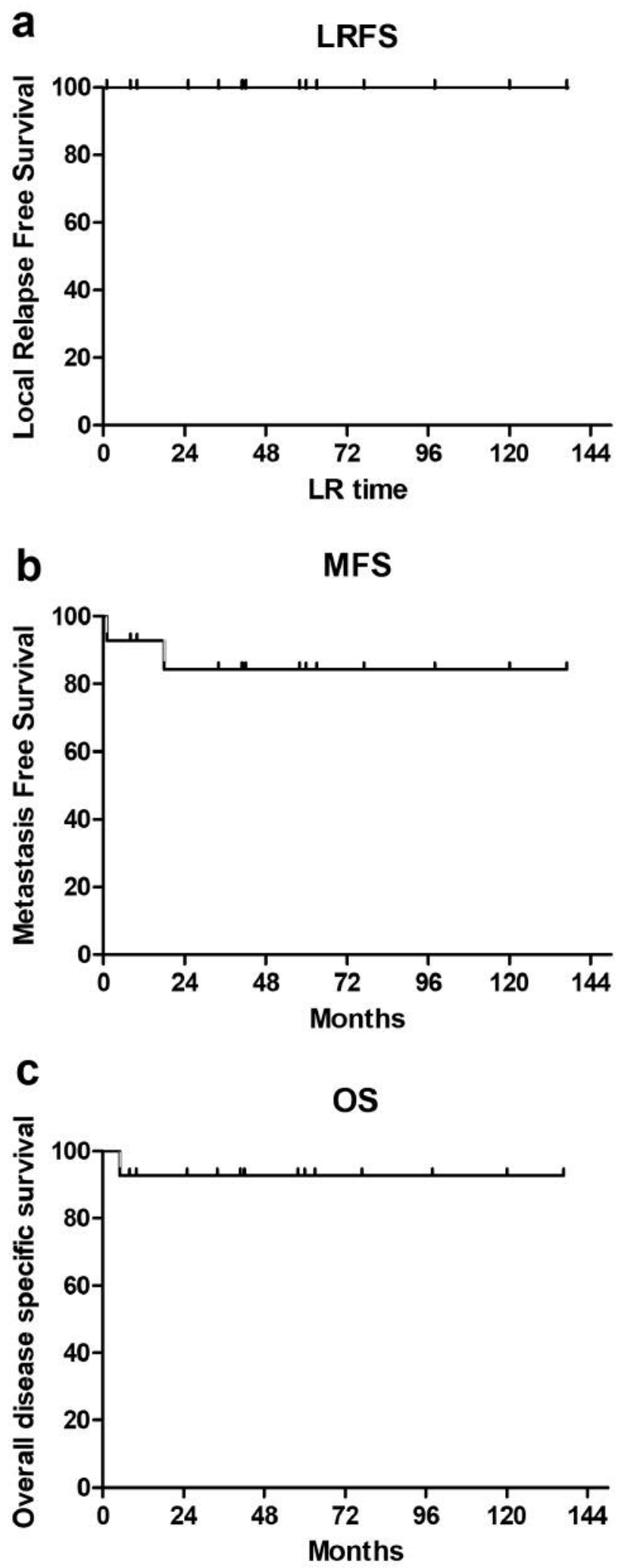

Figure 1. Kaplan-Meier survival curves of (a) local relapse-free survival, (b) metastasis-free survival and (c) overall disease-specific survival. 
patients received chemotherapy. The local relapse-free, distant metastasis-free and disease-specific overall survival Kaplan-Meier curves are shown in Figure 1a, b, c.

\section{Discussion}

Increasing the aggressiveness of the radiotherapy schedule may be useful in the treatment of patients with radioresistant tumors, like sarcomas (17). The usage of large daily fractions (hypofractionation), exceeding the shoulder of cancer cell dose/response curves, may be more effective in eliminating cancer cells with reduced intrinsic radiosensitivity (18) Hypofractionation allows also acceleration of radiotherapy and reduction of the overall treatment time, which may be crucial for the eradication of a subset of tumors undergoing rapid tumor repopulation (19). Combining hypofractionated and accelerated radiotherapy (HypoAR) with chemotherapy offers an aggressive regimen that also exploits the radiosensitizing properties of drugs with proven activity against sarcomas.

In the current study we retrospectively analyzed 14 patients treated with hypofractionated and accelerated radiotherapy. Ten of them received combined concurrent chemo-radiotherapy with cisplatin and liposomal doxorubicin and four of them were treated with radiotherapy alone. Within a median follow-up of 5-years, only two patients developed distant metastasis and one of them is still alive under chemotherapy. None of the patients developed loco-regional recurrence inside the fields of radiotherapy. As sarcomas are considered radio-resistant tumors, the usage of large daily fractions of radiotherapy and the acceleration of the overall treatment time by 11-13 days, compared to a conventionally fractionated (2 Gy/fraction) scheme, may improve the overall radiotherapy efficacy. The administration of cisplatin and liposomal doxorubicin, two active drugs in sarcomas (20), concurrently with radiotherapy may have further improved the radiotherapy efficacy, by sensitizing neoplastic cells to radiation.

It is concluded that concurrent chemo-HypoAR is feasible and provides excellent survival figures. Such regimens deserve testing in randomized trials.

\section{Conflicts of Interest}

There are no conflicts of interest to declare regarding this study.

\section{Authors' Contributions}

SD: Treatment of patients, writing and approval of the manuscript; IMK: Collection of data, statistical analysis, writing and approval of the manuscript; AG: Treatment of patients, writing and approval of the manuscript; MIK: Conception, design, treatment of patients, interpretation of results, drafting and approval of the manuscript.

\section{References}

1 Conklin CM and Longacre TA: Endometrial stromal tumors: the new WHO classification. Adv Anat Pathol 21: 383-393, 2014. PMID: 25299308. DOI: 10.1097/PAP.0000000000000046

2 Gudgeon DH: Leiomyosarcoma of the uterus. Obstet Gynecol 32: 96-100, 1968. PMID: 5742096.

3 Nieminen U and Soderlin E: Sarcoma of the corpus uteri: Results of the treatment of 117 cases. Strahlentherapie 148: 5761, 1974. PMID: 4439433.

4 Wheelock JB, Krebs HB, Schneider V and Goplerud DR: Uterine sarcoma: Analysis of prognostic variables in 71 cases. Am J Obstet Gynecol 151: 1016-1022, 1985. PMID: 2984936. DOI: $10.1016 / 0002-9378(85) 90372-2$

5 DiSaia PJ, Morrow CP, Boronow R, Creasman W and Mittelastaedt L: Endometrial sarcoma: Lymphatic spread pattern. Am J Obstet Gynecol 130: 104-105, 1978. PMID: 619636. DOI: 10.1016/0002-9378(78)90445-3

6 Gokce ZK, Turan T, Karalok A, Tasci T, Ureyen I, Ozkaya E, Kose MF and Tulunay G: Clinical outcomes of uterine carcinosarcoma: results of 94 patients. Int J Gynecol Cancer 25: 279-287, 2015. PMID: 25611900. DOI: 10.1097/IGC.0000000 000000347

7 Menczer J: Review of recommended treatment of uterine carcinosarcoma. Curr Treat Options Oncol 16: 53, 2015. PMID: 26374341. DOI: $10.1007 / \mathrm{s} 11864-015-0370-4$

8 Sahinler I, Atalar B, Tecer GM, Calay Z, Koca S, Atkovar G and Okkan S: Postoperative radiotherapy in the treatment of uterine sarcomas: long-term results and analysis of prognostic factors. J BUON 15: 480-488, 2010. PMID: 20941814.

9 Sorbe B and Johansson B: Prophylactic pelvic irradiation as part of primary therapy in uterine sarcomas. Int J Oncol 32: 11111117, 2008. PMID: 18425339. DOI: 10.3892/ijo.32.5.1111

10 Reed NS, Mangioni C, Malmström H, Scarfone G, Poveda A, Pecorelli S, Tateo S, Franchi M, Jobsen JJ, Coens C, Teodorovic I, Vergote I, Vermorken JB and European Organisation for Research and Treatment of Cancer Gynaecological Cancer Group: Phase III randomised study to evaluate the role of adjuvant pelvic radiotherapy in the treatment of uterine sarcomas stages I and II: an European Organisation for Research and Treatment of Cancer Gynaecological Cancer Group Study (protocol 55874). Eur J Cancer 44: 808-818, 2008. PMID: 18378136. DOI: $10.1016 /$ j.ejca.2008.01.019

11 Mancari R, Signorelli M, Gadducci A, Carinelli S, De Ponti E, Sesana S, Corso S, Chiappa V, Colombo N and Lissoni AA: Adjuvant chemotherapy in stage I-II uterine leiomyosarcoma: a multicentric retrospective study of 140 patients. Gynecol Oncol 133: 531-536, 2014. PMID: 24631454. DOI: 10.1016/j.ygyno. 2014.03.001

12 Pautier P, Floquet A, Gladieff L, Bompas E, Ray-Coquard I, Piperno-Neumann S, Selle F, Guillemet C, Weber B, Largillier R, Bertucci F, Opinel P, Duffaud F, Reynaud-Bougnoux A, Delcambre C, Isambert N, Kerbrat P, Netter-Pinon G, Pinto N, Duvillard P, Haie-Meder C, Lhommé C and Rey A: A randomized clinical trial of adjuvant chemotherapy with doxorubicin, ifosfamide, and cisplatin followed by radiotherapy versus radiotherapy alone in patients with localized uterine sarcomas (SARCGYN study). A study of the French Sarcoma Group. Ann Oncol 24: 1099-1104, 2013. PMID: 23139262. DOI: 10.1093/annonc/mds545 
13 Wolfson AH, Brady MF, Rocereto T, Mannel RS, Lee YC, Futoran RJ, Cohn DE and Ioffe OB: A gynecologic oncology group randomized phase III trial of whole abdominal irradiation (WAI) vs. cisplatin-ifosfamide and mesna (CIM) as post-surgical therapy in stage I-IV carcinosarcoma (CS) of the uterus. Gynecol Oncol 107: 177-185, 2007. PMID: 17822748. DOI: 10.1016/ j.ygyno.2007.07.070

14 Macejewski B, Taylor JM and Wither HR: Alpha/beta and the importance of the size of dose per fraction for late complications in the supraglottic larynx. Radiother Oncol 7: 323-326, 1986. PMID: 3809591. DOI: 10.1016/S0167-8140(86)80061-5

15 Koukourakis MI and Damilakis J: LQ-based model for biological radiotherapy planning. Med Dosim 19: 269-277, 1994. PMID: 7893363.

16 Wong AT, Lee YC, Schwartz D, Lee A, Shao M, Han P, Choi K and Schreiber D: Use of adjuvant chemotherapy, radiation therapy, or combined modality therapy and the impact on survival for uterine carcinosarcoma limited to the pelvis. Int $\mathrm{J}$ Gynecol Cancer 27: 1171-1177, 2017. PMID: 28574930. DOI: 10.1097/IGC.000000000000101

17 Sampo MM, Tuomikoski L, Tarkkanen M, Jääskeläinen AS, Tukiainen EJ, Beule A, Tenhunen M, Böhling TO and Blomqvist CP: Marginal miss or radioresistance? The pattern of local recurrence after operation and $3 \mathrm{D}$ planned radiation treatment in soft tissue sarcoma of the extremities and the limb girdles; an analysis based on image fusion. Acta Oncol 53: 557-562, 2014. PMID: 24199624. DOI: 10.3109/0284186X.2013.844355
18 Bernhard EJ, Stanbridge EJ, Gupta S, Gupta AK, Soto D, Bakanauskas VJ, Cerniglia GJ, Muschel RJ and McKenna WG: Direct evidence for the contribution of activated N-ras and Kras oncogenes to increased intrinsic radiation resistance in human tumor cell lines. Cancer Res 60: 6597-6600, 2000. PMID: 11118040.

19 Jebsen NL, Bruland ØS, Eriksson M, Engellau J, Turesson I, Folin A, Trovik CS and Hall KS: Five-year results from a Scandinavian sarcoma group study (SSG XIII) of adjuvant chemotherapy combined with accelerated radiotherapy in highrisk soft tissue sarcoma of extremities and trunk wall. Int J Radiat Oncol Biol Phys 81: 1359-1366, 2011. PMID: 20933339. DOI: $10.1016 /$ j.ijrobp.2010.07.037

20 Koukourakis MI, Koukouraki S, Giatromanolaki A, Kakolyris S, Georgoulias V, Velidaki A, Archimandritis S and Karkavitsas NN: High intratumoral accumulation of stealth liposomal doxorubicin in sarcomas - rationale for combination with radiotherapy. Acta Oncol 39: 207-211, 2000. PMID: 10859012. DOI: $10.1080 / 028418600430789$
Received June 5, 2019

Revised June 24, 2019

Accepted June 25, 2019 